\title{
Prognostic Value of Angiogenesis and Survivin Expression in Patients with Glioblastoma
}

\author{
Ebru TASTEKIN¹, Vuslat Yurut CALOGLU², Fulya OZ PUYAN', Burcu TOKUC ${ }^{3}$, Murat CALOGLU², \\ Tulin Deniz YALTA ${ }^{1}$, Nuray CAN ${ }^{1}$, Beril GULER ${ }^{1}$ \\ ${ }^{1}$ Trakya University, School of Medicine, Department of Pathology, Edirne, Turkey \\ ${ }^{2}$ Trakya University, School of Medicine, Department of Radiation Oncology, Edirne, Turkey \\ ${ }^{3}$ Trakya University, School of Medicine, Department of Public Health, Edirne, Turkey \\ This study was presented in the $25^{\text {th }}$ National Congress of Pathology and $6^{\text {th }}$ National Congress of Cytopathology (14-17 October 2015, Bursa, Turkey).
}

\section{ABSTRACT}

AIM: Glioblastoma (GBM) is the most common and the most aggressive primary brain tumor with poor prognosis. We aimed to evaluate the association between immunohistochemical expression of survivin and angiogenic parameters (microvessel density and vascular pattern) in patients who underwent surgery for GBM.

MATERIAL and METHODS: The pathology reports and also clinical and follow-up data of patients with GBM were retrospectively evaluated. Control tissues were obtained from the archive for each antibody (Survivin, CD 34). Then, control staining of these antibodies was performed. Vessels were evaluated according to the standardized assessment of vascular pattern.

RESULTS: Mean survival for classical vascular pattern was longer than bizarre vascular pattern $(p<0.001)$. The survival time of patients decreased with increasing score of survivin staining. There was a significant correlation between survivin and survival time $(p<0.001)$. There was no significant correlation between microvessel density and survival time $(p>0.05)$.

CONCLUSION: With these findings, it is considered that high expression of survivin, bizarre vascular pattern and development of secondary GBM correlates with the low survival rates, however microvessel density has no correlation with the survival rates. Since only malignant cells express survivin, it might be a target protein for the development of novel therapies.

KEYWORDS: Glioblastoma, Survivin, Vascular pattern, Survival

\section{INTRODUCTION}

Glioblastoma (GBM) is the most common and the most aggressive primary brain tumor with a poor prognosis (14). The average survival is $12-15$ months, despite advances in chemotherapy, radiotherapy (RT) and surgery $(3,10)$. Due to the tendency of tumor cells to spread rapidly to surrounding tissues, tumor cells can escape from surgical excision. Additionally, because of their increased resistance to apoptosis, they are resistant to radiation and chemotherapy too (7). Both chemotherapy and RT can give rise to deoxyribonucleic acid (DNA) damage but their efficacy is hindered in part by alterations in the cell cycle and apoptotic mechanisms of cancer cells $(27,29)$. Identification of the molecular and genetic mechanisms causing treatment resistance represents an important attempt for the development of more effective therapies against GBMs. The apoptotic mechanism is an important subject for these studies.

Survivin is a member of the inhibitors of apoptosis (IAP) proteins family, and it acts as a key regulator of mitosis, programmed cell death or apoptosis. The role of survivin 
Tastekin E. et al: Angiogenesis and Survivin Expression in GBM

involves the regulation of angiogenesis and chemo-resistance (20). Survivin is normally expressed in fetal tissue but is absent in most of the differentiated adult cells (1). Tumors that highly express survivin generally have an aggressive behavior and poor prognosis and are associated with resistance to RT and chemotherapy (33).

Angiogenesis is defined as the formation of new capillaries from the pre-existing vascular network. Increased angiogenesis is correlated with tumor growth and metastasis in different tumor types (8). Methods for evaluation of angiogenesis including microvessel density (MVD) and vascular pattern have been used in most of the prognostic studies $(9,21)$.

In this study, we aimed to evaluate the association between immunohistochemical expression of survivin and angiogenic parameters (MVD and vascular pattern) in patients who underwent surgery for GBM.

\section{MATERIAL and METHODS}

\section{Patients and Clinicopathological Analysis}

The data of the patients who underwent surgery for a brain tumor between 2000 and 2012 at Trakya University Medical Faculty, and were diagnosed as GBM in the Department of Pathology, were reviewed. The patients, who underwent total tumor excision as confirmed with postoperative radiological examination, were included in this study. The pathology reports and also clinical and follow-up data of the patients were retrospectively evaluated. Tumor slides of all patients were re-evaluated (Figure 1A-D). The local ethics committee approved the study design (Trakya University No.152/201417/10).

\section{Immunohistochemical Analysis}

Control tissues (from data sheets) were obtained from the archive for each antibody. Then, control staining of these

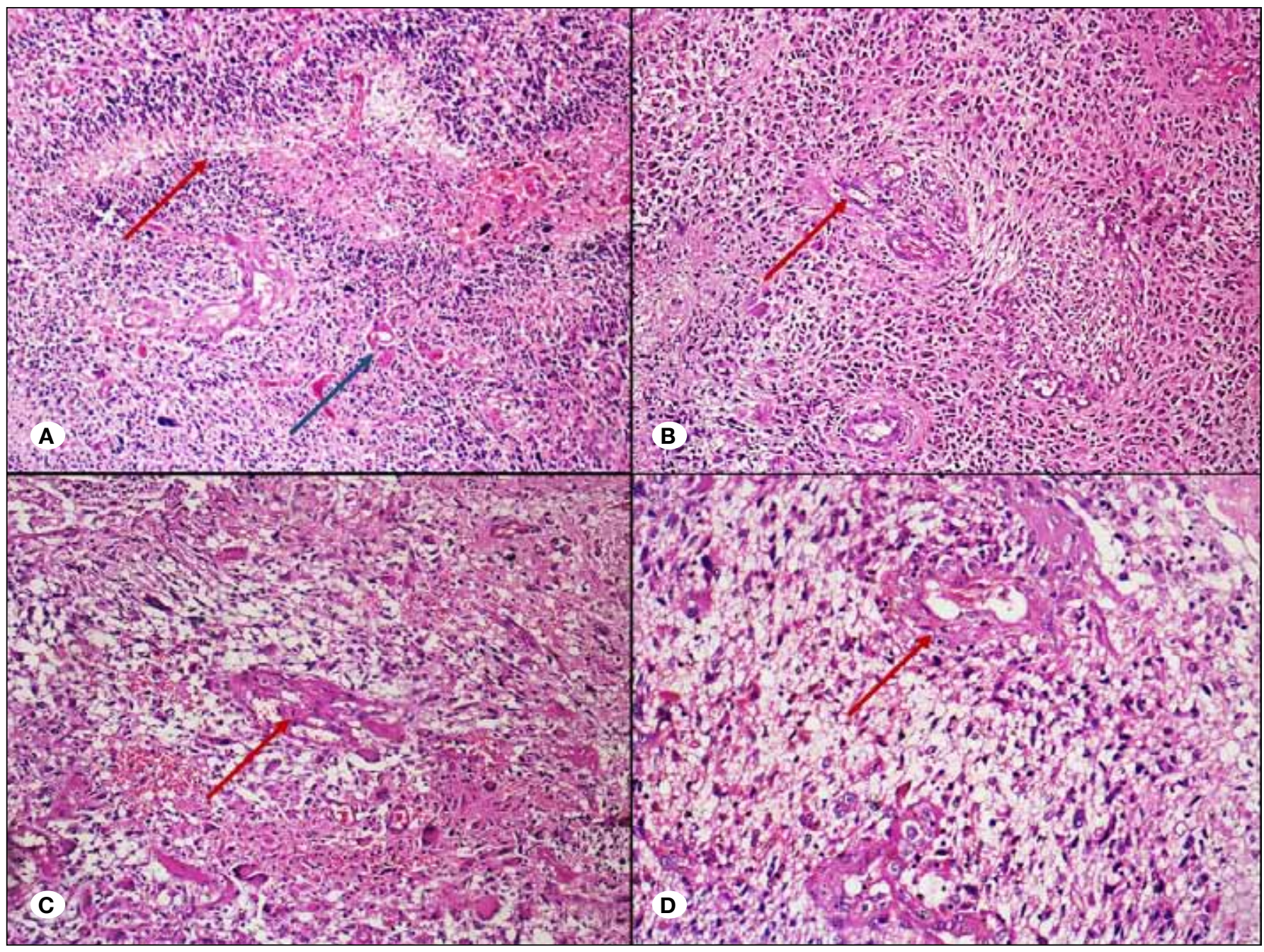

Figure 1: Histopathological features of glioblastoma. Glioblastoma is an aggressive tumor composed of pleomorphic astrocytic cells with marked nuclear atypia and mitosis. A) Palisading necrosis (red arrow) and microvascular proliferation (blue arrow) are important characteristic features of GBM (arrow) (x10). B) Marked nuclear atypia and classical type vascular proliferation (arrow) (x10) C, D) Bizarre vascular pattern with vascular clusters, vascular garlands and glomeruloid vascular formations (arrows)(C:x20, D:x40) (Hematoxylin eosin stain). 
antibodies was performed. Staining for survivin and CD34 antibodies was performed with a fully automated immunohistochemistry and in situ hybridization $(\mathrm{IHC} / \mathrm{ISH})$ staining machine (Ventana BenchMark XT, USA). The following primary antibodies at the indicated dilutions were used for IHC: rabbit polyclonal anti-survivin (NB-500-201 K3, 1:500; Novus Biological Inc., USA) and CD34 (1:100, monoclonal, Neomarkers, USA).

\section{Survivin Evaluation}

For survivin antibody, immunoexpression was based on the percentage of positively stained cells, after counting at least 1000 cells (19). Expression was scored as the following; '-'; if there was no expression, ' + '; if there was expression in $<25$ cells, ' ++ '; if there was expression in $25-50 \%$ cells, ' +++ '; if there was expression in $51-75 \%$ cells, and ' ++++ '; if there was expression in $>75 \%$ cells (32).

\section{MVD Evaluation}

For MVD assessment; areas of tumor containing the highest number of capillaries and small venules were chosen at low power field (40x and $100 \times)$ by light microscopy. Tumors exhibited heterogeneous pattern of MVD, but the areas having the highest neovascularization were found and CD 34 antibody was used to identify the areas of invasive carcinoma with the highest number of discrete microvessels. Any brown-staining endothelial cell or cell cluster that was clearly separate from adjacent microvessels, tumor cells, and other connective tissue elements was considered a single, countable microvessel. Vessel lumens, although usually present, were not necessary for a structure to be defined as a microvessel, and red blood cells were not used to define a vessel lumen. Vessel count was performed on x200 field in five areas and the average value was determined statistically (30).

\section{Vascular Pattern}

Angiogenic subtypes were evaluated on CD34-immunostained tissue sections. Vessels were evaluated according to an algorithm described by Preusser et al. (23). This method was described for the standardized assessment of vascular pattern.

Firstly, the presence of delicate network of branching capillaries throughout the tumor was evaluated as yes/no and was described as score A (not present: 0 , present: 1 ). In the second step, the presence of vascular clusters (score B1), vascular garlands (score B2) or glomeruloid vascular formations (score B3) were evaluated as none: 0 , few/discrete: 1, many/prominent: 2 . Then, score $B$ was calculated as the sum of them (Score B = score B1 + score B2 + score B3). Finally, the vascular pattern was calculated from scores $A$ and $B$ following this algorithm (If score $A=1$ and score $B=2$, then "classic vascular pattern". If score $A=0$ or score $B>2$, then "bizarre vascular pattern").

\section{Statistical Analysis}

Demographic-clinical characteristic of patients were shown as mean \pm standard error of mean (SEM) and percentages. Survival times by clinical stage and differentiation were analyzed by using the Kaplan Meier survival analysis and then the Log-rank test was used for group comparisons. Overall effect of demographic and clinical characteristics that have been found significant effect in Kaplan-Meier survival analysis (survivin, vascular pattern, MVD) was analyzed by the Cox regression model. $\mathrm{P}$ value $<0.05$ considered statistically significant. Statistical analysis was performed by using the SPSS 20.0 software (IBM Corp. Released 2011. IBM SPSS Statistics for Windows, Version 20.0. Armonk, NY: IBM Corp.).

\section{RESULTS}

Eighty patients who had been diagnosed as GBM were included in the study. Forty-five of the patients were male and 35 were female $(56.2 \%$ and $43.8 \%)$. The average age was $58.55 \pm 12.82$ years (21-87 years). Five $(5 \%)$ of the patients were alive during the study. Thirty tumors $(37.5 \%)$ were localized in the temporal lobe, $28(35 \%)$ tumors were in the frontal lobe, $19(23.8 \%)$ tumors were in the parietal lobe and 3 (3.8\%) tumors were in the occipital lobe. Seventy-five (93.8\%) of the tumors were primary and $5(6.2 \%)$ were secondary (Table I).

Bizarre vascular pattern was present in $40(50 \%)$ and classical vascular pattern was present in $40(50 \%)$ cases (Figure 1A-D) (Table I). While survival time was $5.11 \pm 0.72$ months in tumors with bizarre vascular pattern, it was $18.76 \pm 2.68$ months in patients with classical vascular pattern. Mean survival for classical vascular pattern was longer than bizarre vascular pattern. This finding was statistically significant $(p<0.001)$ (Figure 2A, B) (Table II).

Survivin immunohistochemical $(\mathrm{IHC})$ expression was observed in all tumors. There was no unstained case. Survivin immunostaining was mainly in the nucleus of tumor cells associated with cytoplasmic staining in some cases. All cases exhibited positive staining with survivin antibody (Figure 3A-D) (Table I). IHC score was 1 in 15 cases (18.8\%), 2 in 21 cases (26.3\%), 3 in 20 cases (25\%) and 4 in 24 cases (30\%). There was a significant difference between survival times of survivin IHC groups $(p<0.001)$ (Figure 2A, B). And there was a significant negative correlation between survivin staining and survival time, with the survival time of patients decreasing with increasing score of survivin staining $(r=-0.656, p=0.000)$ (Table II).

Patients were classified in two groups based on MVD. Group 1 consisted of tumors with MVD count $<70 \%$, group 2 consisted of tumors with MVD $\geq 70 \%$. Mean survival time for group 1 was $12.00 \pm 3.26$ months and for group 2 it was $11.616 \pm 1.31$ months. There was no significant difference between survival time of groups ( $p>0.05)$. There was no significant correlation between MVD and survival time $(r=-0.490, p=0.667)$. There was also no significant correlation between survivin IHC expression and MVD ( $r=0.023, p=0.843)$ (Table II).

Patients with secondary GBM had longer survival time than primary GBM and this difference was statically significant $(p=0.048)$. There was no relationship between tumor location and survival $(p>0.05)$. There was also no significant correlation between survival times and gender $(p=0.68)$. 
Overall effect of demographic and clinical characteristics that have been found to have a significant effect on survival time in Kaplan-Meier survival analysis (survivin, vascular pattern, MVD) was evaluated by Cox regression model. In the Cox regression analysis, we found nearly two times higher mortality risk (HR=1.975 (95\% Cl $1.020-3.823), p=.044)$ in bizarre vascular pattern and nearly 80 times higher mortality

Table I: Clinical and Histopathological Characteristics of Patients with GBM

\begin{tabular}{lll}
\hline Characteristic & \multicolumn{2}{l}{} \\
\hline \multirow{2}{*}{ Gender } & Female & $35(43.8 \%)$ \\
\cline { 2 - 3 } & Male & $45(56.2 \%)$ \\
\hline \multirow{3}{*}{ Localization } & Temporal & $30(37.5 \%)$ \\
\cline { 2 - 3 } & Frontal & $28(35.0 \%)$ \\
\cline { 2 - 3 } & Parietal & $19(23.8 \%)$ \\
\cline { 2 - 3 } & Occipital & $3(3.8 \%)$ \\
\hline \multirow{2}{*}{ Primary/Secondary } & Primary & $75(93.8 \%)$ \\
\cline { 2 - 3 } Vascular pattern & Secondary & $5(6.2 \%)$ \\
\hline & 1 & $40(50 \%)$ \\
\cline { 2 - 3 } & 2 & $40(50 \%)$ \\
\hline \multirow{3}{*}{ Survivin } & 1 & $15(18.8 \%)$ \\
\cline { 2 - 3 } & 2 & $21(26.3 \%)$ \\
\hline \multirow{2}{*}{ MVD } & 3 & $20(25.0 \%)$ \\
\hline
\end{tabular}

MVD: Microvessel density. risk in survivin IHC score group 4 to group 1 (HR=79.008 $(95 \%$ Cl $17.719-352.299), p=.000$ ) (Table III).

\section{DISCUSSION}

Glioblastoma is classified as a grade IV tumor by the World Health Organization (WHO) (18). GBMs are known by their aggressive and infiltrating nature. The most important problem in the management of GBM treatment is intrinsic or acquired resistance to treatment $(18,25,35)$. New and more effective agents are very important. In this study, we demonstrated that GBMs have high expression rates of survivin and the

Table II: The Results of the Kaplan-Meier Survival Analysis

\begin{tabular}{|c|c|c|c|}
\hline & & $\begin{array}{c}\text { Mean survival time } \\
\text { (month) } \\
\text { Mean } \pm \text { SEM }\end{array}$ & $\mathbf{p}$ \\
\hline \multirow{2}{*}{ Vascular pattern } & 1 & $5.109 \pm 0.72$ & \multirow{2}{*}{0.000} \\
\hline & 2 & $18.758 \pm 2.62$ & \\
\hline \multirow{4}{*}{ Survivin } & 1 & $28.595 \pm 5.12$ & \multirow{4}{*}{0.000} \\
\hline & 2 & $14.500 \pm 1.54$ & \\
\hline & 3 & $5.474 \pm 0.32$ & \\
\hline & 4 & $3.500 \pm 0.30$ & \\
\hline \multirow{2}{*}{ MVD } & $<70$ & $12.000 \pm 3.26$ & \multirow{2}{*}{0.648} \\
\hline & $\geq 70$ & $11.616 \pm 1.31$ & \\
\hline \multirow{2}{*}{$\begin{array}{l}\text { Primary/ } \\
\text { Secondary }\end{array}$} & Primary & $10.864 \pm 1.50$ & \multirow{2}{*}{0.048} \\
\hline & Secondary & $26.600 \pm 9.14$ & \\
\hline \multirow{2}{*}{ Gender } & Male & $12.166 \pm 1.86$ & \multirow{2}{*}{0.684} \\
\hline & Female & $11.884 \pm 2.78$ & \\
\hline
\end{tabular}

SEM: Standard error of mean, MVD: Microvessel density.

Table III: Overall Effect of Survivin Positivity and Angiogenic Features on Survival

\section{Cox Regression-Univariate}

\begin{tabular}{|c|c|c|c|c|}
\hline & & HR & $95 \% \mathrm{Cl}$ for $\mathrm{HR}$ & $\mathbf{p}$ \\
\hline \multirow{2}{*}{ Vascular pattern } & Classical & \multicolumn{3}{|c|}{ reference } \\
\hline & Bizarre & 1.975 & $1.020-3.823$ & 0.044 \\
\hline \multirow{4}{*}{ Survivin } & 1 & \multicolumn{3}{|c|}{ reference } \\
\hline & 2 & 2.702 & $1.179-6.256$ & .019 \\
\hline & 3 & 30.487 & $7.078-131.320$ & .000 \\
\hline & 4 & 79.008 & $17.719-352.299$ & .000 \\
\hline \multirow{2}{*}{ Primary/Secondary } & Primary & \multicolumn{3}{|c|}{ reference } \\
\hline & Secondary & 0.525 & $0.192-1.437$ & .201 \\
\hline
\end{tabular}

HR: Hazard ratio. 


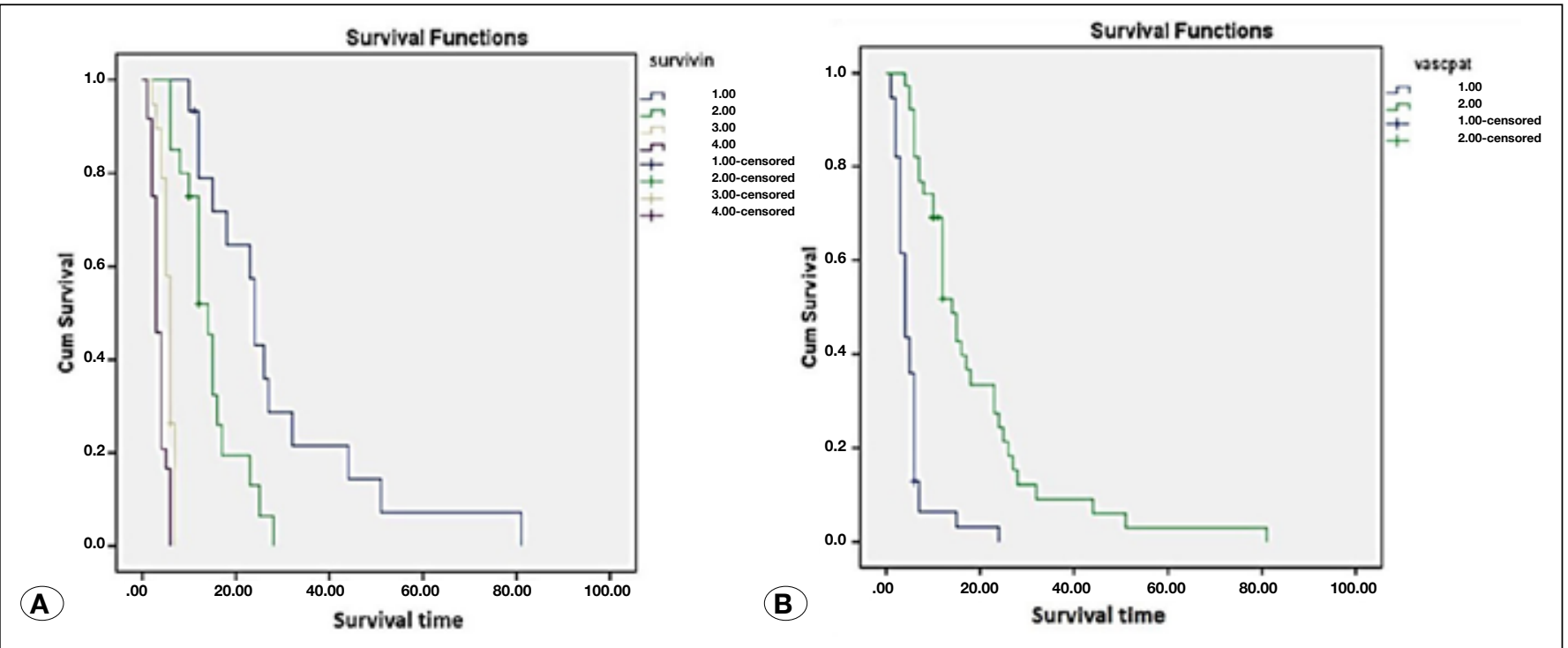

Figure 2: A) Relationship between survivin and survival, B) relationship between vascular pattern (vascpat) and survival.

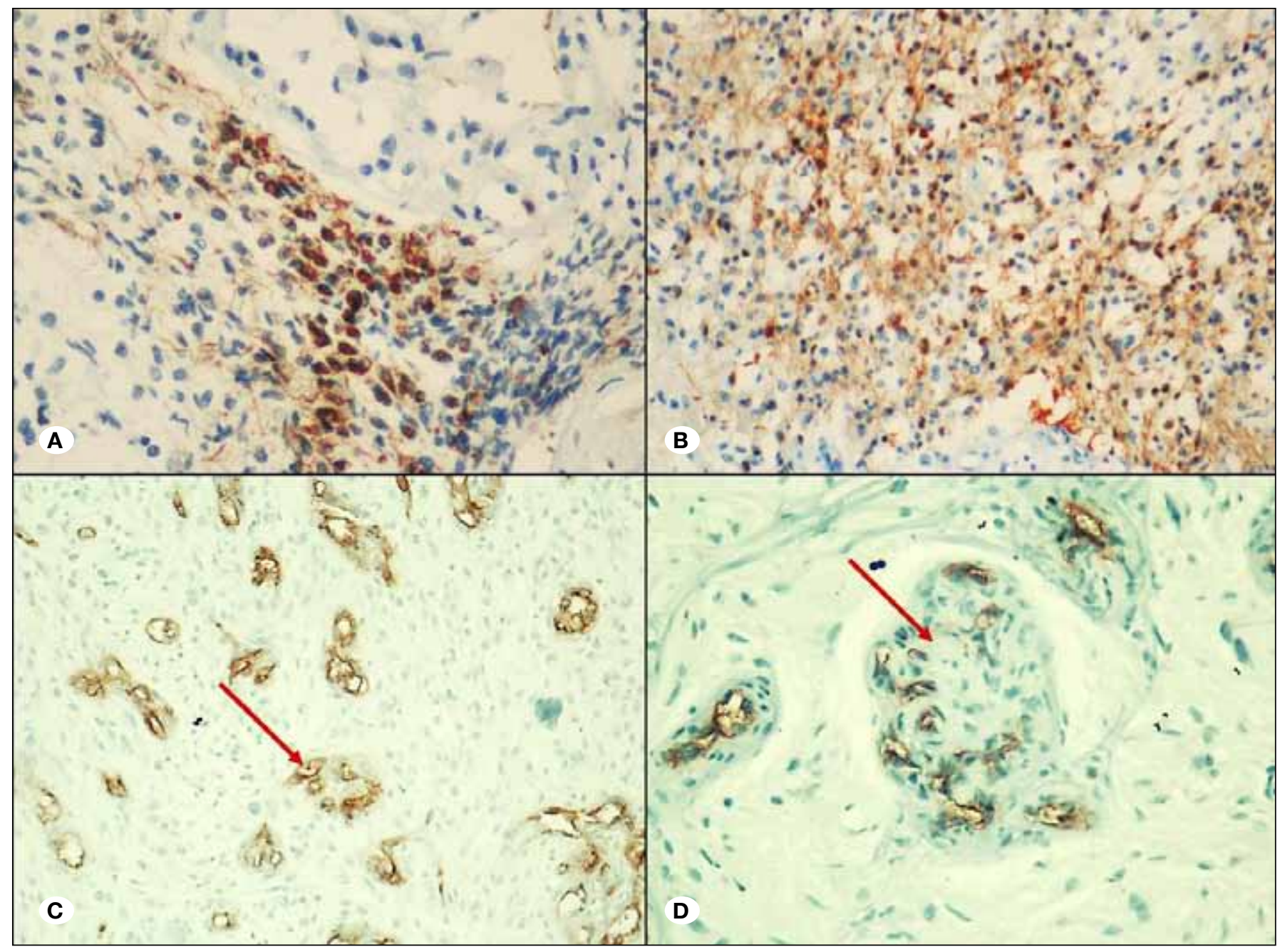

Figure 3: A, B) Survivin positivity in glioblastoma cells (A. Nuclear staining, x50; B. Cytoplasmic staining, x50). C, D) CD34 staining is showing vascular features of GBM (C. Microvascular density evaluation, arrow: small-sized vessels, x50; D. Vascular pattern evaluation, arrow: bizarre vascular pattern with glomeruloid vessels, x50). 
percentage of immunoreactive cells had a strong inverse association with the survival of patients. Bizarre vascular pattern was related with poor prognosis.

Survivin is a member of IAPs. Survivin is known to be normally highly expressed in fetal tissue but absent in normal adult cells (1). It is expressed extensively in a lot of human cancers $(1,16)$ and the increased expression of survivin appears to be associated with the poor prognosis and aggressive behavior of the tumors. GBM is an example of these tumors. Especially, the survivin expression is correlated with the survival of patients. Some authors have reported increased cytoplasmic expression in human gliomas. The degree of immunoexpression of survivin in gliomas was correlated with the increase in the pathological grade of tumor (11, 26). In addition, patients who had been diagnosed as GBM, with detectable survivin expression, have been observed to have significantly shorter survival time (6). Based on these results, it can be suggested that survivin might be involved in tumorigenesis and progression of GBM (32). So, survivin would be useful to predict the prognosis and it may be a target gene for the new therapeutic agents. Hence, survivin inhibition might enable the elimination of GBM without affecting normal tissue $(2,17,34)$.

Some studies showed a relationship between survivin expression and radiation resistance in some malignancies. Chakravarti et al. (6) reported that survivin suppresses radiation-induced cell death because it improves doublestrand DNA break repair in tumor cells. Sasaki et al. (26) showed that suppression of survivin by small interfering ribonucleic acid (RNA)(siRNA) improved radiosensitivity of tumor cells. Based on these findings, anti-survivin therapies may enhance the radiation response, and be correlated with better results $(12,13,26)$.

GBM has been a major candidate for anti-angiogenic treatment because of its prominent neovascularization (22). Some studies have found a close relation between MVD and prognosis of GBMs $(5,28,31)$. The vascular pattern may also have an important role on the efficacy of anti-angiogenic therapy. Evaluation of the microvascular features of GBMs can help enable the use of anti-angiogenic drugs $(4,5)$. However, there are no validated drugs that can be used to determine the angiogenic features of GBM in relation to anti-angiogenic therapies. There is also no data on the threat of the angiogenic part of the tumor (24).

In our study, results of survivin staining supported that survivin was highly expressed in GBMs. Survivin expression was seen in tumors without a significant relationship with MVD. The difference of survivin expression between the bizarre and classical vascular patterns in GBMs was statistically significant.

A few studies showed that bizarre vascular pattern was an independent prognostic factor in GBMs. Bernsen et al. (4) showed that the presence of a low amount of bizarre vascular pattern with a high amount of classical vascular pattern together in GBM was an independent factor for high survival rate. Also, Preusser et al. (24) reported that the more vascular pattern was an independent prognostic factor in GBMs. They stated that while the bizarre vascular pattern was related with shorter survival times, the classic vascular pattern was related with higher survival rates. There were statistically significant results for vascular patterns in our study, similar to the previous results.

Sharma et al. (28) showed that increased angiogenesis was characterized with an increased number of disproportionate size and irregular vessels in GBMs. They did not show relationship between angiogenic features and proliferation. Even though they observed glomeruloid proliferation in GBMs, their study's follow-up data were not adequate for precise results (15). Recently Preusser et al. (24) evaluated the influence of MVD and vascular patterns on GBM patients' survival. In this study, inter-observer differences were high in choosing the hot spot area. They also repeatedly assessed MVD and vascular patterns in two GBM series. They showed that there was no association between MVD, vascular patterns and patient outcomes. They concluded that inter-observer difference was a limiting factor for the clinical utility of hot-spot MVD and vascular patterns as prognostic factors in GBMs.

Onguru et al. (21) stated that these methods were questionable, because of regional heterogeneity in the vascularization of GBMs. We agree with Onguru et al. and we thought that the MVD was an independent factor for prognosis. It can be evaluated in only vascular areas of partial sampled tumors and this condition may affect the histopathological evaluation of tumor because of the tumor heterogeneity and inadequate specimen. Therefore, only totally resected tumors were included in our study.

De novo GBMs have similar aggressive features. The special glomeruloid vascular pattern was more effective on survival than MVD. The special histopathological vascular features of GBM and methodological differences can also affect these results. We thought that our findings might represent an important effect on patient management. Our study showed that there is no association between MVD and patient's clinical outcome. However, new studies with stereological or neuroradiological methods should be planned with large series.

\section{CONCLUSION}

Survivin expression and vascular pattern are related with survival time of patients with GBM. This relationship was not detected with MVD and survival of the patients. Survivin may be an ideal target for cancer therapy to kill tumor cells alone and leave the normal cells unaffected. Suppression of survivin with powerful anticancer drugs could be an effective treatment modality for glioblastomas.

\section{- REFERENCES}

1. Adida C, Crotty PL, McGrath J, Berrebi D, Diebold J, Altieri DC: Developmentally regulated expression of the novel cancer anti-apoptosis gene survivin in human and mouse differentiation. Am J Pathol 152(1):43-49, 1998

2. Altieri DC: Survivin, cancer networks and pathway-directed drug discovery. Nat Rev Cancer 8(1):61-70, 2008 
Tastekin E. et al: Angiogenesis and Survivin Expression in GBM

3. Benitez JA, Dominguez-Monzon G, Segovia J: Conventiona and gene therapy strategies for the treatment of brain tumors. Curr Med Chem 15(8):729-742, 2008

4. Bernsen HJJA, Rijken PF, Hagemeier NE, van der Koge AJ: A quantitative analysis of vascularization and perfusion of human glioma xenografts at different implantation sites. Microvasc Res 57(3):244-257, 1999

5. Birner P, Piribauer M, Fischer I, Gatterbauer B, Marosi C Ambros PF, Ambros IM, Bredel M, Oberhuber G, Rossler $\mathrm{K}$, Budka $\mathrm{H}$, Harris AL, Hainfellner JA: Vascular patterns in glioblastoma influence clinical outcome and associate with variable expression of angiogenic proteins: Evidence for distinct angiogenic subtypes. Brain Pathol 13(2):133-143, 2003

6. Chakravarti A, Noll E, Black PM, Finkelstein DF, Finkelstein DM, Dyson NJ, Loeffler JS: Quantitatively determined survivin expression levels are of prognostic value in human gliomas. J Clin Oncol 20(4):1063-1068, 2002

7. Drappatz J, Norden AD, Wen PY: Therapeutic strategies for inhibiting invasion in glioblastoma. Expert Rev Neurother 9(4):519-534, 2009

8. Folkman $\mathrm{J}$ : What is the evidence that tumors are angiogenesis dependent? J Natl Cancer Inst 82(1):4-6, 1990

9. Hansen S, Grabau DA, Rose C, Bak M, Sorensen FB: Angiogenesis in breast cancer: A comparative study of the observer variability of methods for determining microvessel density. Lab Invest 78(12):1563-1573, 1998

10. Henson JW: Treatment of glioblastoma multiforme: A new standard. Arch Neurol 63(3):337-341, 2006

11. Jiao BH, Yao ZG, Geng SM, Zuo SH: Expression of survivin, a novel apoptosis inhibitor and cell cycle regulatory protein, in human gliomas. Chin Med J (Engl) 117(4):612-614, 2004

12. Kanwar JR, Kamalapuram SK, Kanwar RK: Targeting survivin in cancer: Patent review. Expert Opin Ther Pat 20(12):17231737, 2010

13. Kanwar RK, Cheung CH, Chang JY, Kanwar JR: Recent advances in anti-survivin treatments for cancer. Curr Med Chem 17(15):1509-1515, 2010

14. Khasraw M, Lassman AB: Advances in the treatment of malignant gliomas. Curr Oncol Rep 12(1):26-33, 2010

15. Lakka SS, Rao JS: Antiangiogenic therapy in brain tumors. Expert Rev Neurother 8(10):1457-1473, 2008

16. Li F, Ambrosini G, Chu EY, Plescia J, Tognin S, Marchisio PC, Altieri DC: Control of apoptosis and mitotic spindle checkpoint by survivin. Nature 396(6711):580-584, 1998

17. Liao MH, Lin WC, Wen HC, Pu HF: Tithonia diversifolia and its main active component tagitinin $\mathrm{C}$ induce survivin inhibition and $\mathrm{G} 2 / \mathrm{M}$ arrest in human malignant glioblastoma cells. Fitoterapia 82(3):331-341, 2011

18. Louis DN, Ohgaki H, Wiestler OD, Cavenee WK, Burger PC, Jouvet A, Scheithauer BW, Kleihues P: The 2007 WHO classification of tumours of the central nervous system. Acta Neuropathol 114(2):97-109, 2007

19. Mellai M, Caldera V, Patrucco A, Annovazzi L, Schiffer D: Survivin expression in glioblastomas correlates with proliferation, but not with apoptosis. Anticancer Res 28(1a):109-118, 2008
20. Mita AC, Mita MM, Nawrocki ST, Giles FJ: Survivin: Key regulator of mitosis and apoptosis and novel target for cancer therapeutics. Clin Cancer Res 14(16):5000-5005, 2008

21. Onguru O, Gamsizkan M, Ulutin C, Gunhan O: Cyclooxygenase-2 (Cox-2) expression and angiogenesis in glioblastoma. Neuropathology 28(1):29-34, 2008

22. Plate $\mathrm{KH}$ : Gene therapy of malignant glioma via inhibition of tumor angiogenesis. Cancer Metastasis Rev 15(2):237-240, 1996

23. Preusser $M$, Birner $P$, Hainfellner JA: Algorithm for the standardized assessment of vascular patterns in glioblastoma specimens. Clin Neuropathol 23(5):238-240, 2004

24. Preusser M, Heinzl H, Gelpi E, Schonegger K, Haberler C, Birner P, Marosi C, Hegi M, Gorlia T, Hainfellner JA, European Organization for R, Treatment of Cancer Brain Tumor G: Histopathologic assessment of hot-spot microvessel density and vascular patterns in glioblastoma: Poor observer agreement limits clinical utility as prognostic factors: A translational research project of the European Organization for Research and Treatment of Cancer Brain Tumor Group. Cancer 107(1):162-170, 2006

25. Sarkaria JN, Kitange GJ, James CD, Plummer R, Calvert $\mathrm{H}$, Weller $\mathrm{M}$, Wick W: Mechanisms of chemoresistance to alkylating agents in malignant glioma. Clin Cancer Res 14(10):2900-2908, 2008

26. Sasaki T, Lopes MB, Hankins GR, Helm GA: Expression of survivin, an inhibitor of apoptosis protein, in tumors of the nervous system. Acta Neuropathol 104(1):105-109, 2002

27. Shah MA, Schwartz GK: Cell cycle-mediated drug resistance: An emerging concept in cancer therapy. Clin Cancer Res 7(8):2168-2181, 2001

28. Sharma S, Sharma MC, Gupta DK, Sarkar C: Angiogenic patterns and their quantitation in high grade astrocytic tumors. J Neurooncol 79(1):19-30, 2006

29. Verheij M, Bartelink H: Radiation-induced apoptosis. Cell Tissue Res 301(1):133-142, 2000

30. Weidner N, Semple JP, Welch WR, Folkman J: Tumor angiogenesis and metastasis-correlation in invasive breast carcinoma. N Engl J Med 324(1):1-8, 1991

31. Wesseling P, Ruiter DJ, Burger PC: Angiogenesis in brain tumors; Pathobiological and clinical aspects. J Neurooncol 32(3):253-265, 1997

32. Xie D, Zeng YX, Wang HJ, Wen JM, Tao Y, Sham JS, Guan XY: Expression of cytoplasmic and nuclear Survivin in primary and secondary human glioblastoma. $\mathrm{Br} \mathrm{J}$ Cancer 94(1):108-114, 2006

33. Yamamoto H, Ngan CY, Monden M: Cancer cells survive with survivin. Cancer Sci 99(9):1709-1714, 2008

34. Zhonghong L, Lianjie L, Changqing Z, Ying H, Yu J, Yan L: The influence of survivin shRNA on the cell cycle and the invasion of SW480 cells of colorectal carcinoma. J Exp Clin Cancer Res 27(1):20, 2008

35. Ziegler DS, Wright RD, Kesari S, Lemieux ME, Tran MA, Jain M, Zawel L, Kung AL: Resistance of human glioblastoma multiforme cells to growth factor inhibitors is overcome by blockade of inhibitor of apoptosis proteins. J Clin Invest 118(9):3109-3122, 2008 